Didáctica. Lengua y literatura

ISSN: 1130-0531

http://dx.doi.org/10.5209/DIDA.57134

\title{
Estudio autobiográfico sobre la problemática de un maestro novel de inglés durante el desarrollo de las destrezas escritas de su alumnado
}

\author{
Javier González Jiménez ${ }^{1}$
}

Recibido: 22 de junio de 2016 / Aceptado: 9 de junio de 2017

Resumen En este artículo se presenta un estudio de caso desde una perspectiva autobiográfica en el que, a través del análisis de los diarios de aula de un profesor novel de inglés de la etapa de Educación Primaria, se ahonda en el conocimiento de los problemas prácticos y dilemas que le surgen al desarrollar en sus alumnos las destrezas escritas. Partiendo del análisis cualitativo de las reflexiones realizadas en dichos documentos personales se ha elaborado un informe narrativo para llegar a comprender mejor el proceso de pensamiento y las actuaciones del novel en su práctica docente para desarrollar la lectoescritura en la lengua extranjera.

Palabras clave: enseñanza de lenguas; lenguas extranjeras; destrezas escritas; maestro novel; diario de clase, solución de problemas.

\section{[en] Autobiographical study on the problems of a beginning teacher of English in developing writing skills of pupils}

\begin{abstract}
This paper presents an autobiographical self-study that, through the analysis of a Primary Education beginning teacher's class diaries, delves into the knowledge of the practical problems and dilemmas that appear in developing the pupils' writing skills. Based on the qualitative analysis of the reflections included in these personal documents, a narrative report has been written in order to better understand the beginning teacher's cognitive process and actions during daily practice to develop literacy in a foreign language.

Key words: Language teaching; foreign languages; written skills; beginning teacher; classroom diary, problem solving.
\end{abstract}

\section{[fr] Étude autobiographique sur la problématique d'un enseignant d'anglais débutant au cours du le développement des compétences écrites de ses élèves}

Résumé. Cet article présente une étude de cas autobiographique qui essaie de connaître les problèmes pratiques et les dilemmes d'un professeur d'anglais de l'enseignement primaire au cours du le développement des compétences écrites de leur élèves. Sur la base de l'analyse qualitative de ses réflexions un rapport a été écrit pour obtenir une meilleure compréhension du processus de pensée et les actions de la pratique de l'enseignement du professeur pour développer la lecture et l'écriture dans la langue étrangère.

\footnotetext{
Departamento de Didáctica de la Lengua y la Literatura

Facultad de Educación y Trabajo Social

Universidad de Valladolid

javiergj@dlyl.uva.es
} 
Mots-clés: l'enseignement des langues; les langues étrangères; les compétences écrites; enseignant novice, journal de classe; solution de problèmes.

Sumario: 1. Introducción. 2. Objetivos del estudio. 3. Metodología. 4. Resultados. 5. Discusión. 6. Bibliografía.

Cómo citar: González Jiménez, J. (2017). Estudio autobiográfico sobre la problemática de un maestro novel de inglés durante el desarrollo de las destrezas escritas de su alumnado. Didáctica. Lengua y literatura, 29, 2017, 139-154.

\section{Introducción}

Las investigaciones relativas al pensamiento docente, enmarcadas en el Paradigma Mediacional centrado en el Profesor, tienen la dificultad de centrarse en un objeto de estudio altamente subjetivo, complejo y de difícil acceso. Sin embargo, la gran utilidad de la información obtenida de cara a la comprensión del proceso de enseñanza-aprendizaje ha hecho que la corriente de investigación relativa al pensamiento del profesor sea una referencia dentro de dicho Paradigma.

En el caso concreto de los estudios relativos al pensamiento del profesor novel, y especialmente aquellos que ponen el centro de atención en los problemas y toma de decisiones, están permitiendo conocer de primera mano cómo se desarrolla el periodo de iniciación profesional como referencia para la mejora de la formación inicial.

Son muchas las investigaciones sobre este objeto de estudio, sin embargo, las relativas específicamente al pensamiento del profesor novel de inglés se reducen drásticamente. Teniendo en cuenta la revisión llevada a cabo por Borg (2006), la mayor parte de las investigaciones sobre la problemática y los procesos de pensamiento del profesorado de lenguas se han realizado a partir de la segunda mitad de la década de los noventa y tomando como referente profesorado con experiencia docente. $\mathrm{Al}$ mismo tiempo, gran parte de los estudios se han realizado en países donde el inglés es lengua materna con lo que los resultados no son extrapolables al profesorado de inglés como lengua extranjera. A esto hay que añadir que aunque existe una amplia tradición investigadora sobre la problemática de los profesores noveles como colectivo, la mayoría de estudios se centra en problemas compartidos por docentes sin adentrarse apenas en las peculiaridades de cada área de enseñanza, tal como queda reflejado en la revisión bibliográfica realizada por Veenman $(1984,1988)$. En esta línea, en nuestro país se tienen como referente estudios como los de Vera (1988), González, Álvarez y González (1991) y Marcelo (1993).

Más recientemente, el interés por conocer qué tipos de problemas comparten los docentes, la importancia que los otorgan o la frecuencia de aparición, está dejando paso al interés por conocer los procesos de pensamiento singulares de cada profesor generados en contextos concretos y ante problemas específicos de su área. El objetivo de estas investigaciones ya no es la generalización de los resultados buscando grandes categorías de problemas comunes a la mayoría de contextos, sino más bien cómo cada docente, de manera individual e irrepetible procesa la información en cada contexto problemático y qué decisiones toma y a qué recurre para superar las dificultades que se le presentan. 
En este sentido, la finalidad del estudio descrito en el presente artículo no es la simple identificación de la problemática del profesorado novel de inglés a la hora de trabajar las destrezas escritas en el aula ni la generalización de resultados causales. Es adentrarse en su pensamiento y en la toma de decisiones interactivas para contribuir a la construcción de un conocimiento compartido que mediante reflexión genere un aprendizaje realista (Esteve, Melief y Alsina, 2010) y una mayor comprensión de la realidad descrita, que derive en la construcción de un conocimiento didáctico sobre y desde la práctica para volver a ella y mejorarla.

\section{Objetivos del estudio}

En concreto, el propósito de esta investigación es describir y comprender el pensamiento y acción de un profesor novel de inglés durante el periodo de iniciación profesional ante las situaciones problemáticas de su práctica diaria a la hora de trabajar las destrezas escritas en el aula. De este propósito derivan los siguientes objetivos de investigación:

1. Reducir el vacío de información que actualmente existe dentro de los estudios sobre la problemática del profesor novel en relación al área de Lengua Extranjera (inglés).

2. Lograr una mayor comprensión del periodo de iniciación profesional de los profesores de inglés, así como de los agentes, factores y elementos del contexto educativo que pueden influir en su desarrollo.

3. Identificar, describir y analizar las situaciones problemáticas, dilemas y dificultades específicas que se encuentra un profesor novel de inglés en el desarrollo de las destrezas escritas en su alumnado.

4. Contribuir a una mayor comprensión de los procesos cognitivos y pensamientos que genera el profesor novel ante dichas situaciones problemáticas en conexión con las acciones, decisiones y respuestas propias que es capaz de elaborar para solucionarlas.

5. Crear oportunidades para activar y desarrollar el pensamiento reflexivo y crítico en el profesorado novel con el fin de favorecer su crecimiento profesional.

\section{Metodología}

Los procesos de pensamiento del profesor como objeto de estudio generan no pocas dificultades debido a su alta subjetividad y dificultad de acceso a lo que una persona está pensando en un momento concreto. Es por tanto necesario en este tipo de investigaciones optar por un enfoque sensible a esta circunstancia si realmente queremos captar la complejidad de la realidad educativa y del pensamiento del docente. En consecuencia, esta investigación se encuadra en una perspectiva fenomenológica, interpretativa-naturalista, característica de los estudios etnográficos cualitativos, donde la narración es la opción más apropiada para manejar y representar la información en un estudio de caso de estas características (Arnaus, 1995). La naturaleza del estudio lleva a optar, en consecuencia, por un método 
inductivo, superando el tradicional debate entre la investigación positivista y la interpretativa-naturalista.

Más concretamente, teniendo en cuenta las aportaciones de autores como Bolívar (2001, 2002) y Sandín (2003), este estudio se enmarca dentro de la investigación (auto)biográfica-narrativa, en donde es el propio docente quien relata en primera persona la realidad que está experimentando. Lo que se pretende, según Esteve et al. (2008) es interpretar una realidad educativa desde una perspectiva émica, holística y contextualizada, lo que nos lleva a optar como estrategia metodológica óptima por un estudio de caso en un nivel microcontextual. Esta opción metodológica permite desarrollar un proceso de indagación para llegar a comprender de forma sistemática y en profundidad una realidad social como la educativa (Sandín, 2003).

Dentro de este marco metodológico, el presente estudio se ha llevado a cabo a partir del análisis de los diarios personales de un profesor novel de inglés, funcionario de carrera, con una experiencia docente de dos cursos académicos en dos centros educativos públicos de Educación Infantil y Primaria de Valladolid. Dichos documentos escritos fueron elaborados durante los cursos escolares 2002-2003 y 2003-2004.

La investigación se desarrolló a lo largo de tres fases diferenciadas: fase de descripción extensiva del pensamiento docente, fase de categorización y análisis de la información previa y fase de reconstrucción narrativa.

a) Primera fase de descripción extensiva: la redacción de los diarios del profesor.

En esta primera fase el profesor novel fue registrando de manera inmediata y sistemática, simultáneamente a un proceso constante de auto-observación y reflexión, las situaciones problemáticas en el momento y contexto en el que se producían. Estos registros permitieron reflejar de manera lo más fiel posible la realidad y el pensamiento docente, además de actuar posteriormente como activadores del recuerdo para el profesor a la hora de escribir los dos diarios.

Estos documentos personales, teniendo en cuenta la tipología establecida por Plummer (1989) y Zabalza (1991), se pueden catalogar como diarios autobiográficos mixtos, presentando una secuencia clara de lo general a lo concreto y de lo descriptivo a la reflexión como sugieren Porlán y Martín (1991). Inicialmente dichos diarios presentan una dimensión descriptiva de la dinámica general del aula desde una perspectiva global con múltiples referencias al contexto. Progresivamente se adentran en la descripción detallada de las dificultades y problemas prácticos con una mayor presencia de valoraciones e interpretaciones de la realidad. A continuación, se inicia la dimensión subjetiva que supone la interpretación de esos problemas prácticos haciendo explícito no solo el pensamiento del profesor mediante reflexión, sino también sus sentimientos, pensamientos y valores. La activación durante el proceso de escritura de los diarios de un proceso de "metarreflexión" (Melief et al., 2010), activó a su vez un proceso cíclico y retroalimentado en el que las reflexiones daban lugar a un nuevo proceso de toma de decisiones interactivas.

b) Segunda fase de análisis y categorización de los diarios.

La segunda fase del proceso de investigación abarca el análisis de los datos. Mediante un primer análisis retardado mediante reflexión, se identificaron una serie de macrocategorías de situaciones consideradas como problemáticas por el docente, destacando los "Problemas en la aplicación de estrategias de enseñanza y en el 
aprendizaje de los alumnos", que es donde se agrupan los problemas relacionados con el desarrollo de las destrezas escritas. Esta y otras subcategorías se identificaron en una segunda lectura más detenida de los documentos personales durante la que se llevó a cabo un análisis categórico del contenido buscando relaciones y regularidades. Este análisis más concreto se realizó utilizando el software QDA “Qualrus".

c) Tercera fase de elaboración del informe de investigación.

En esta última fase se reorganizó la información recogida en las distintas categorías mediante un informe, con una dimensión tanto descriptiva como reflexiva, para facilitar al lector la comprensión de la problemática que encuentra el profesor novel y los factores condicionantes en su aparición e intensidad. Teniendo en cuenta las aportaciones de Bolívar (2002), la primera dimensión surge de un análisis de los datos narrativos una vez categorizados, que de una forma objetiva y con cierta distancia recoge la problemática así como el contenido del pensamiento y las acciones que generan. La segunda dimensión del informe es la que recopila mediante citas ilustrativas amplias el proceso de reflexión sobre la realidad vivida por el profesor novel.

\section{Resultados}

El análisis categórico de los documentos personales del profesor novel no solamente ha permitido la identificación y clasificación de las situaciones problemáticas, sino que además ha sido el referente para llevar a cabo una reconstrucción narrativa, con extractos de los diarios de aula, en la que se describen de una manera más profunda las principales dificultades, dilemas prácticos y toma de decisiones durante el proceso de desarrollo de la competencia comunicativa escrita de los alumnos.

\section{Informe narrativo a partir de los diarios personales: problemas en el desarrollo de las destrezas escritas en el alumnado.}

Dentro de la descripción de las dificultades asociadas al desarrollo de las destrezas en el aula de inglés, uno de los grupos de problemas más recurrentes es el que se refiere al desarrollo de la destreza de Reading. El primer problema al que se enfrenta el profesor novel es la escasa importancia que en su opinión se le da a esta destreza en las guías didácticas frente a la producción escrita. Lo observa tanto en el número como en el tipo de actividades produciéndose un claro desequilibrio en el tratamiento de estas destrezas que intenta compensar mediante actividades extra, como la transcripción para su lectura de los input orales, la lectura grupal en voz alta o el préstamo de libros en inglés para su lectura en casa.

Con respecto a la clase de $5^{\circ}$, aunque también me pasa en $6^{\circ}$, me he dado cuenta de que la destreza básica que tienen menos desarrollada es el Reading. Estos niños escriben mejor que leen, cuando se supone que se debería desarrollar antes la destreza receptiva que al mismo tiempo es cognitivamente más sencilla ya que supone reconocer. En cambio el Writing requiere un conocimiento básico de la estructura gramatical, del spelling, etc. Esto parece indicar que se ha puesto más empeño o se le ha dado más importancia a la destreza productiva que a la receptiva. Esto suele 
pasar entre los profesores, y a mí también me pasa, ya que lo que el alumno produce es sinónimo de aprendizaje, es más fácil de evaluar, y además, en el caso de la escritura permanece. Esto nos hace volcarnos en la producción, hasta el punto de que en la mayoría de los exámenes y actividades de carácter evaluativo prima la escritura sobre el resto de destrezas. Esto es algo que me preocupa y que estoy intentando evitar. Todas las historias del libro que solo están destinadas al Listening las estoy transcribiendo y se las facilito después de haberlas comprendido. Llevo ya varios días escribiendo esos diálogos y pequeñas historias en la pizarra intercalando el Listening y el Reading, ya que en estas guías no sé por qué pero apenas proponen actividades de lectura, todo es escribir, especialmente en el activity book (2 de noviembre de 2002).

Por otro lado, el profesor novel se encuentra principalmente con dificultades a la hora de que los alumnos asocien la ortografía de las palabras con su pronunciación dentro de un sistema ortográfico muy irregular en comparación con la lengua materna. Las escasas reglas ortográficas, llenas de excepciones, unidas al deseo de respetar el pensamiento inductivo de los alumnos, hacen que el docente opte por la memoria visual como principal medio de aprendizaje a través de actividades controladas.

Lo que el profesor novel hace es asociar la forma escrita de las palabras y frases con su pronunciación casi de forma simultánea cuando las presenta aunque prefiere que los alumnos interioricen primero la forma oral para evitar una lectura directa en donde los niños, sobre todo los más pequeños, asocien cada grafema con un sonido.

Creo que es necesario que comiencen a identificar ciertas palabras escritas una vez que hemos trabajado su dimensión oral. Al principio del año pasado tenía mucho cuidado con esto, hasta que el niño no asimilaba la forma oral de las palabras no introducía la forma escrita. El problema que surgía es que las sesiones se alargaban demasiado y los niños se aburrían del tópico aunque los juegos y actividades fueran diferentes. En este sentido, cada vez más estoy intercalando la presentación del vocabulario nuevo en su forma oral y escrita. Como los niños leen las palabras tal cual, partiendo de su lengua materna, siempre he tenido miedo de que se afiance si el niño no tenía interiorizada la forma oral, pero me estoy dando cuenta de que no hay ningún problema, de que se puede presentar simultáneamente $(21$ de noviembre de 2002).

En esta asociación cobra especial importancia el apoyo visual con el que presenta las nuevas palabras utilizando actividades de flashcards. El profesor novel entiende que esta dificultad de los niños para reconocer la relación grafema-fonema le lleva a dar más importancia a la lectura en voz alta con una correcta pronunciación que a la comprensión lectora, sin embargo, a la hora de evaluar, esta última es más sencilla y lleva menos tiempo que ir leyendo en voz alta un texto con toda la clase corrigiendo los errores de pronunciación.

Con respecto a este último aspecto, de nuevo una corrección inmediata y constante puede generar en el alumno un bloqueo y un rechazo a la lectura, pero al mismo tiempo suavizar la corrección puede generar, en opinión del profesor novel, errores de pronunciación y una asociación fonema-grafema incorrecta que se puede afianzar en el alumno. La solución que se plantea ante este dilema es leer el texto mientras los alumnos lo siguen en silencio estableciéndolo como modelo, trabajar mediante 
juegos orales y ortográficos las palabras nuevas o más complicadas, repetir la frase o palabra mal pronunciada una vez que el alumno termine su turno o comentar al final de la lectura errores comunes, siempre evitando interrumpir la lectura lo que aumentaría el filtro afectivo de los niños.

Después de dar lengua a primera hora he bajado a la clase de $3^{\circ} \mathrm{A}$. La actividad central de hoy ha sido el comic del libro que cierra cada lección manteniendo los mismos personajes. Esta actividad les encanta porque conecta con sus intereses y su vida diaria y sobre todo porque demuestran un gran interés en leer en inglés, igual que en escribir. (...) Es posible que esta predisposición tenga mucho que ver con mi esfuerzo por evitar la corrección del error de manera directa tratando los fallos de pronunciación como algo a erradicar. En su lugar y gracias a los principios que adquirimos durante la formación inicial que nos alejan de caer en las prácticas tradicionales, lo que hago una vez escuchado y leído por mí todo seguido es que lean viñeta por viñeta la cual vuelvo a repetir proporcionándoles el input correcto de manera inmediata. Lo hago poco a poco porque es justo cuando cometen el error cuando en mi opinión se les debe ofrecer el modelo adecuado ya que si no es menos eficaz (15 de marzo de 2004).

Por otro lado, al igual que en el caso de la destreza de Listening, el profesor novel pretende establecer una rutina de actuación a la hora de llevar a cabo actividades de lectura, ya que un proceso claro de actuación evita la aparición de muchos problemas. Uno de ellos es el desequilibrio en el que inconscientemente puede caer a la hora de trabajar las diferentes sub-destrezas, principalmente la comprensión de la idea global de un texto escrito y la extracción de ideas secundarias y datos específicos. Un ejemplo de este tipo de problemas es la obsesión de los alumnos por entender todas las palabras de un texto escrito incluso cayendo en la traducción. Para evitar esta tendencia que hace la lectura muy pesada y estresante para los alumnos el profesor novel intenta desarrollar en los niños la capacidad de deducir e inferir el significado de palabras desconocidas por el sentido de la frase, evitando en los primeros niveles el uso del diccionario, y dando más importancia a la extracción inicial de la idea general.

Otra medida que he tomado ha sido facilitar a los dos niños más destacados de la clase un librito de inglés, más o menos de su nivel, para que lo lean en casa. Según me han dicho les cuesta mucho entender todas las palabras. Para desarrollar una lectura comprensiva les he dicho que tiene que entender el significado general y que sólo busquen las palabras más importantes, porque si no se aburren y lo dejan (2 de noviembre de 2002).

Poco a poco este año están utilizando con soltura el diccionario que se ha convertido en una herramienta más de las clases de inglés. Creo que les he enseñado a utilizarlo sin caer en el error de la dependencia que yo experimenté cuando iba al colegio y que suponía la incapacidad percibida de entender un texto si no buscaba cada palabra desconocida en el diccionario, o bien la tendencia a utilizarlo cuando quería escribir un texto literalmente después de haberlo pensado en español. Creo que esto lo he evitado porque solo lo utilizamos cuando realmente perjudica la expresión o la comprensión facilitando el desarrollo de otras capacidades esenciales como inferir significados por el contexto (22 de marzo de 2004). 
En ciclos superiores el profesor novel también pretende desarrollar la capacidad de reconocer cómo está organizado un texto y las estrategias utilizadas para darle coherencia y cohesión. Este conocimiento permite al alumno buscar la información más rápido y reconocer la función del texto, para lo que el profesor novel trabaja la lectura apoyándose en la identificación de la estructura de cartas, noticias, poemas, cuentos, etc., antes de que los alumnos produzcan textos similares centrándose en el proceso más que el producto.

Aunque lo tenía preparado para la siguiente sesión, al acabar la actividad de elaboración de la lista tan pronto, pasé a exponer la estructura de una carta para que pudiesen escribir la suya a los Reyes Magos en inglés. Esta idea les ha encantado y han realizado bastantes sugerencias sobre qué debía contener la carta (saludo, peticiones, felicitación, deseos, despedida, posdata...) y de qué manera. Esto me ha permitido trabajar aspectos como los saludos y despedidas formales, la estructura de una carta..., algo bastante importante para el desarrollo de la destreza del Writing (17 de diciembre de 2003).

El proceso de desarrollo de la lectura que sigue el profesor novel es muy similar al de Listening, empezando por un momento inicial de preparación de la lectura. En esta primera fase tiene como objetivo motivar a los alumnos, presentar el texto que van a leer y los contenidos clave para la comprensión activando los conocimientos previos de los alumnos, principalmente repasando vocabulario y estructuras ya conocidas y que aparecen en el texto.

He leído despacio el texto pidiéndoles que intentasen adivinar por el contexto y las fotos el significado de las palabras nuevas acertando bastantes. El resto ha sido a base de pistas y gestos, todo ello para evitar la traducción del texto, ni siquiera parte de él porque si no se acostumbran mal. (...) Por último, a modo de resumen y para ir presentando el vocabulario, les he pedido que buscasen en el texto los distintos departamentos que puede tener un gran almacén realizando un esquema en la pizarra (7 de mayo de 2004).

Tras la presentación pide a los alumnos que hagan predicciones sobre el contenido del texto partiendo por ejemplo del título, de las primeras líneas o del apoyo visual, con el fin de aumentar su deseo de leer y comprobar dichas predicciones. Finalmente y antes de comenzar las actividades relacionadas con la lectura el profesor novel informa a sus alumnos sobre el tipo de lectura, si es intensiva o extensiva, su propósito, y las destrezas específicas y técnicas que deben poner en funcionamiento para tener éxito en la comprensión y extracción de información de cara a las actividades posteriores. Para el docente esta primera fase es fundamental y va más allá de la mera motivación ya que permite que los alumnos, normalmente con poca autonomía lectora, vayan tomando conciencia de la importancia de elegir la estrategia adecuada según la finalidad y el tipo de texto.

En un segundo momento que coincide con la propia lectura, los alumnos realizan un primer acercamiento al texto extrayendo las ideas principales. En una segunda lectura con un mayor grado de atención extraen las ideas secundarias y obtienen datos específicos siguiendo las claves de búsqueda que da el docente, anotando la información si es necesario para las actividades posteriores. El profesor novel des- 
cribe la rutina que ha desarrollado en la realización de las actividades de lectura de la siguiente manera:

Lo que hago es leer primero el texto entero para que capten con la ayuda visual la idea más general, después leo párrafo por párrafo para que se fijen en la pronunciación y luego leen ellos volviendo a ofrecer el input correcto al final de sus lecturas para no interrumpirles. Después de cada lectura parcial intento que entre todos deduzcan el significado esencial. De esta manera logro que mejoren la pronunciación y la comprensión pero sé que es algo momentáneo y que para que lo interioricen van a necesitar más actividades de lectura. Finalmente, y aunque no lo hago a menudo hemos traducido los textos después de desarrollar la comprensión de los mismos. No es un traducción literal, sino que reproducen lo que ellos entienden al leer en inglés en voz alta y creo que es efectivo (enero 2003).

Esta rutina de actuación finaliza con un tercer momento, posterior a la lectura. Tras varias lecturas al menos una de ellas en voz alta y otra en silencio, el profesor novel pregunta a los alumnos una serie de cuestiones sobre lo que han leído para apoyar la comprensión general y qué tipo de información específica han extraído asegurando así el éxito de las actividades asociadas. En el caso de que el grado de comprensión no haya sido suficiente propone una nueva lectura pero reforzando aún más el proceso, por ejemplo con más apoyo visual, parando en los momentos clave o enfatizando la lectura que él mismo hace del texto. Tras las lecturas propone una serie de actividades controladas con el fin de afianzar el reconocimiento de la ortografía de las palabras y la asociación con su pronunciación.

A continuación, el profesor novel propone una serie de actividades más creativas en las que los alumnos deben utilizar la información obtenida con una funcionalidad concreta. Es el momento en el que el profesor novel integra la destreza de Reading con las productivas Speaking y Writing, por ejemplo realizar un role play después de la lectura y modificar un diálogo o añadir nuevos versos a un poema o a una canción.

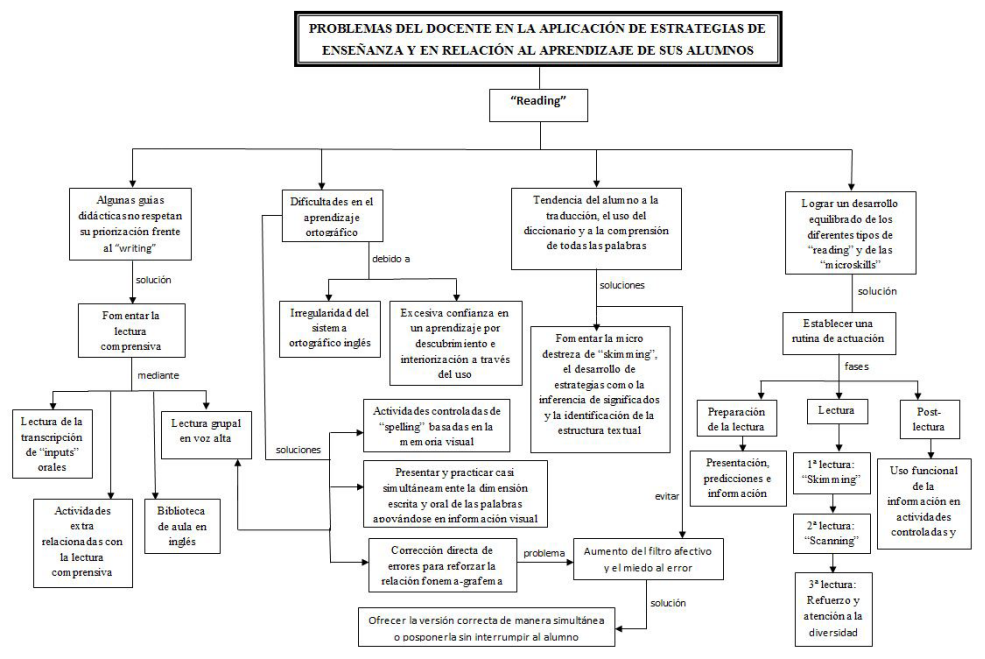

Figura 1. Mapa conceptual problemas prácticos y dilemas respecto al Reading 
Finalmente, en el análisis categórico de los diarios se puede identificar otro grupo de dificultades relativas al desarrollo de la destreza de Writing. El profesor novel es consciente de que esta es una de las destrezas más complicadas de adquirir y por tanto de enseñar desde el principio dado su alto grado de complejidad desde el punto de vista cognitivo. Para llegar a una producción escrita más o menos autónoma y creativa, se requiere un conocimiento amplio de léxico, ortografía, estructuras gramaticales, etc. así como en niveles superiores la capacidad para organizar e integrar la información con coherencia dependiendo de la estructura y función del texto escrito. Esta es la razón por la que el profesor novel tiene especial cuidado en introducir de forma repentina esta destreza en el primer ciclo cuando los alumnos aún están en el proceso de desarrollo inicial de la lectoescritura en la lengua materna. En este sentido, y de nuevo debido principalmente a la irregularidad ortográfica de la lengua inglesa, el profesor novel tiene la inquietud de que se produzcan interferencias entre ambas lenguas. Para ello no introduce la dimensión escrita hasta que los alumnos tienen muy afianzada la oral y reconocen visualmente las palabras, aunque esto supone cierto aburrimiento por los deseos de los alumnos de escribir y una falta posterior de tiempo para trabajar la escritura. La solución que se plantea es intercalar ambas dimensiones más rápido aunque no se haya afianzado del todo la pronunciación ni el reconocimiento visual ya que la práctica posterior soluciona posibles errores.

Suelo respetar la prioridad de las actividades orales, es decir, primero trabajamos un tema desde estas destrezas, aunque haya referencias escritas sobre todo para trabajar el spelling del vocabulario nuevo, y una vez que compruebo que dominan el tema pasamos a la versión escrita. Hay en mi opinión varias razones para respetar este orden, principalmente el hecho de que cognitivamente la expresión escrita es más complicada que la oral, aunque también se pueden producir interferencias, sobre todo en ciclos inferiores donde los niños tienden a leer lo que está escrito como si leyeran en español porque no han interiorizado la pronunciación y escriben tal cual les suena. Después, una vez que pronuncian correctamente y reconocen visualmente las palabras es más fácil que se dé la asociación con la dimensión escrita evitando errores que podrían consolidarse (9 de junio de 2004).

En consecuencia, para respetar al máximo la capacidad del alumno en edades tempranas, el profesor novel no trabaja la dimensión escrita en Infantil y lo hace en el primer ciclo sólo a nivel de palabra y frases muy sencillas basadas en un modelo de referencia. En cambio en la destreza productiva oral la transición de la práctica controlada a la producción más libre y creativa es más rápida, ya que los alumnos disponen de destrezas adquiridas en el aprendizaje de la lengua materna que pueden transferir al inglés mostrándose más sueltos al lidiar con una destreza más sencilla desde un punto de vista cognitivo. Realmente es la complejidad de la tarea y la poca experiencia de los alumnos más pequeños lo que hace que el profesor novel tenga especial cuidado a la hora de llevar a cabo esa transición en lo que respecta al Writing. De forma gradual el profesor novel comienza con la escritura correcta de palabras y frases sencillas, muy trabajadas oralmente, mediante juegos y actividades controladas como por ejemplo: "labelling pictures", "ordering words and simple sentences", "listing words", "writing notes and cards", etc. Más que la corrección ortográfica lo 
que le preocupa al docente es de nuevo la conexión que establece el alumno entre el significado de la palabra, su dimensión oral y escrita.

Me gusta primero trabajar el vocabulario básico oralmente, utilizando si es posible realia y cuando está consolidado lo escriben debajo de cada dibujo, así confeccionan una ficha de referencia para cualquier consulta que van interiorizando visualmente. Cuando la terminen de confeccionar y hayan consolidado el vocabulario básico es cuando seguimos con el libro, las Listening, actividades de Writing..., con mayores garantías de éxito, ya que considero que en estos niveles los campos semánticos son la base de la comprensión y el desarrollo de las destrezas orales y escritas (25 de septiembre de 2003).

En una segunda fase o nivel de desarrollo, que normalmente suele coincidir con el segundo ciclo, aunque depende de la capacidad de los alumnos, el objetivo es que estos pasen de escribir frases a escribir párrafos sencillos como por ejemplo al hacer una pequeña descripción, completar un diálogo, añadir una nueva estrofa a una canción, etc. Aunque en esta fase el profesor novel aumenta significativamente las actividades y el tiempo de escritura, los textos que producen los alumnos son todavía muy cortos y dirigidos, y en los que se les da la estructura básica. La posibilidad de expresión libre es aún muy reducida, con lo que toman especial importancia el uso de técnicas didácticas como el parallel writing planteándolo como una solución intermedia.

A la clase de $4^{\circ}$ la he propuesto realizar un proyecto sobre los medios de transporte. Este proyecto no forma parte de la unidad didáctica del libro, ya que es una manera de introducir ciertos contenidos y lograr objetivos adicionales que me interesan. El libro de texto es muy limitado y se centra en el aprendizaje del vocabulario y en pequeñas estructuras. Lo que voy a hacer es sustituir actividades repetitivas y poco aprovechables por una serie de proyectos. En este en cuestión los niños deben recortar de revistas fotografías de medios de transportes que vamos a describir en un mural utilizando adjetivos contrarios (fast/slow, old/new, cheap/expensive...), colores, formas..., junto con las estructuras lingüísticas necesarias. De esta manera logro que los niños estén motivados y vean un resultado real, físico, de su aprendizaje (28 de noviembre de 2002).

Finalmente, en el tercer ciclo el objetivo que se propone el profesor novel es que los alumnos escriban textos de forma autónoma, con corrección ortográfica y gramatical, pero sobre todo haciendo hincapié en aspectos como la coherencia del texto, la organización y claridad de ideas, la adecuación de la estructura al propósito y contexto, el estilo, etc. Es el momento de que los alumnos reflexionen sobre el propio proceso de escritura aplicando estrategias con autonomía para lo que les propone un gran variedad de textos para escribir (cartas, noticias, poemas, narración de experiencias personales, pequeños cuentos, diálogos...) con diferentes grados de formalidad, registro y estructuras donde aplican sus conocimientos gramaticales y lingüísticos de manera contextualizada y además desarrollan su creatividad y autonomía.

Antes del recreo he dado clase a $6^{\circ} \mathrm{C}$. Seguimos con el tema de las vacaciones pero hoy se me ha ocurrido una actividad bastante interesante aunque puede parecer ob- 
via. Les he propuesto escribir una postal de verdad en inglés sobre sus vacaciones pasadas y mandársela a alguien de clase, un amigo, etc. Con esto no sólo consigo que apliquen todos los contenidos trabajados de manera más o menos autónoma, sino que se den cuenta de que el inglés es útil y puede formar parte de su vida real, que no se queda en el aula. Les ha encantado la idea y hemos comenzado a realizar el borrador que después corrijo antes de la versión final. Esto del borrador lo aplico por la asignatura de Didáctica del Inglés en la que se daba importancia a este paso intermedio como una actividad más de Writing y de evaluación planteando muchas actividades asociadas. La verdad es que es muy útil para que reflexionen sobre la estructura, el tipo de vocabulario a emplear..., y sobre todo que vean que puede haber varias posibilidades de expresar las mismas ideas, lo que se logra con la co-evaluación del borrador (21 de mayo de 2004).

De nuevo, el profesor novel vuelve a encontrarse con un problema ya descrito en la destreza de Speaking, que es cómo hacer compatible una producción escrita autónoma, creativa y motivante en la que los contenidos lingüísticos estén al servicio de su deseo de comunicar con sus verdaderas capacidades y conocimiento lingüístico. La solución a esta cuestión le viene dada por parte de sus alumnos:

(...) ha surgido un problema que no esperaba y que es propio de este tipo de actividades tan cercanas a sus intereses y es el conflicto que se produce entre lo que verdaderamente interesa al niño y las necesidades lingüísticas y comunicativas que le surgen y que pueden no coincidir con su capacidad ni con los contenidos que hemos trabajado. Lo digo por una niña que quería elegir como deporte la equitación porque lo practica, otra la natación..., pero son deportes que a la hora de describir lo que eres capaz de hacer o no, requieren o bien mucho vocabulario que no hemos manejado o apenas dan juego a la hora de hablar de ellos a este nivel. Por lo tanto me ha surgido el dilema de si dar respuesta a sus verdaderas motivaciones o hacer que se ajusten a un trabajo cercano a lo trabajado en el aula y que me permita evaluar su progreso. O lo que es lo mismo me he tenido que plantear si el objetivo del project es puramente evaluativo o bien es una oportunidad para desarrollar su capacidad de expresión escrita de manera más libre y creativa como resultado de un proceso de investigación (uso de diccionario, ayuda del profesor, búsqueda en Internet...). La solución ha sido más sencilla de lo que esperaba y me la ha dado la propia niña: hacer dos proyectos, uno del deporte que practica (lo que la costará mucho y seguramente tenga más carencias y fallos) dando respuesta a sus intereses y otro de un deporte de los trabajados en clase dando respuesta a mis objetivos evaluativos, de consolidación y de síntesis (8 de marzo de 2004).

En síntesis, el profesor novel lleva a cabo dos procesos de enseñanza-aprendizaje: uno inicial centrado en el producto en donde la corrección gramatical y ortográfica es lo más importante, corrigiendo los errores de manera sistemática en un contexto controlado; otro, centrado en el proceso, en donde lo fundamental es el desarrollo de la sub-competencia discursiva y la capacidad de los alumnos para reflexionar sobre el texto más adecuado teniendo en cuenta los posibles lectores y el contexto.

Con respecto al primero, el proceso centrado en el producto, al profesor novel le surge un dilema. Por un lado el mayor peso e importancia de las destrezas orales hace que dedique un mayor número de actividades y tiempo de la sesión al desarrollo de 
estas dejando poco tiempo para trabajar la escritura. Esto implica que no se dé en el aula una práctica de Writing tan extensa y rica como para que los alumnos hayan interiorizado, a través del uso aspectos como el spelling de las palabras o la estructura correcta de las frases. Esto hace que el proceso de aprendizaje sea más lento y que surjan los primeros problemas generando en el profesor novel la inquietud de no estar dedicando el tiempo suficiente a esta destreza.

(...) me preocupa bastante la destreza del Writing y sobre todo los errores de spelling. Son capaces de reconocer los contenidos y expresarlos, pero cometen muchos errores al escribir lo que indica que no los han interiorizado dentro de esta destreza productiva. Para comprobar lo que me temía les hice un pequeño dictado a partir de flashcards para que ellos dijesen cada palabra y después la escribieran, además les pedí que expresaran por escrito si les gustaba o no ciertos deportes, hobbies y comidas. El resultado ha sido bastantes errores de spelling, lo que indica que no se ha trabajado lo suficiente, pero el problema básico es que no dispongo de actividades de ampliación y refuerzo sobre estos temas a estos niveles ( 8 de octubre de 2003).

Aunque no es generalizado yo no sé si estos errores se deben a una falta de práctica, de atención o a otras razones que se me escapan pero me preocupan. En cambio en la estructuración de las oraciones y en el dominio oral del vocabulario no se equivocan, es decir, tienen las descripciones bastante bien, excepto estos errores de spelling (24 de marzo de 2004).

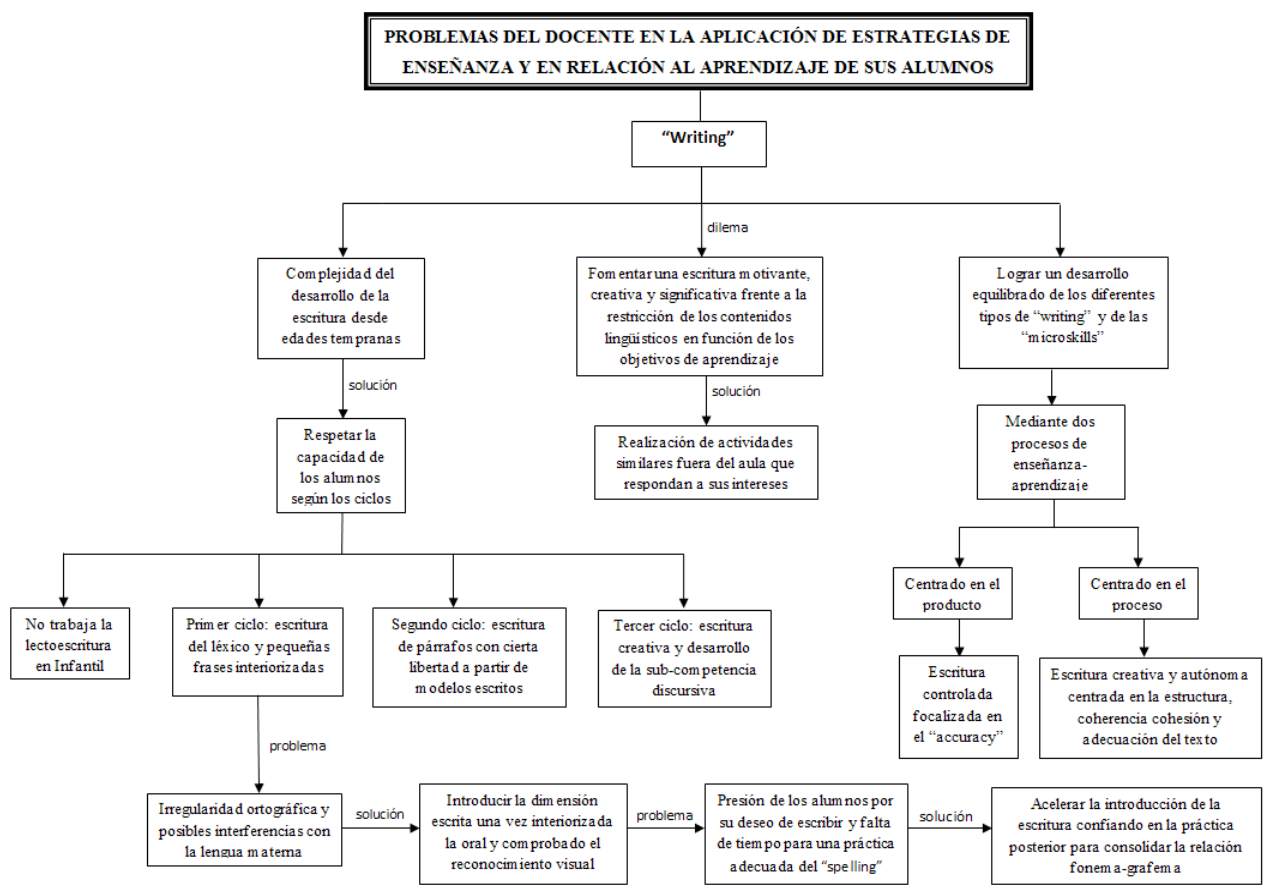

Figura 2. Mapa conceptual problemas prácticos y dilemas respecto al Writing 


\section{Discusión}

A pesar de que existe una larga tradición investigadora dentro del Paradigma del Pensamiento del Profesor, en el caso concreto del profesorado de las áreas lingüísticas hay pocos estudios sobre sus procesos de pensamiento, especialmente si tenemos en cuenta la especial importancia que se está dando a los nuevos contextos y situaciones plurilingües en las escuelas europeas (Cambra y Palou, 2007).

La presencia de diferentes lenguas y culturas en las aulas está generando contextos de enseñanza y aprendizaje cada vez más complejos que requieren una constante revisión y actualización de los principios y teorías de la Didáctica de las Lenguas Extranjeras. Este conocimiento teórico debe retroalimentarse constantemente con el conocimiento práctico generado por los docentes en la realidad del aula, porque es en esos contextos donde se ponen a prueba los principios y teorías didácticas.

De entre todas las fases que pueden componer la vida profesional de un docente, es probablemente el periodo de iniciación profesional el que más información nos aporte al respecto ya que es cuando el profesor intenta aplicar los conocimientos adquiridos durante la formación inicial de manera más directa ante la falta de un conocimiento práctico que le darán los años de experiencia. Así que es de gran utilidad la información recogida en los diarios de aula que suelen describir con precisión la realidad educativa. Este material descriptivo tan valioso puede ser la base para numerosas investigaciones y utilizarse durante la formación inicial, especialmente en el periodo de prácticas, como activador de la reflexión en los futuros docentes, tal como afirma Borg (2001): "Teachers' stories of their experience - communicated through diaries, journals, autobiographies and other forms of narrative - provide insight into what being a teacher means which is instructive for other teachers, teacher educators and researchers" (172).

Sería muy interesante que los planes de formación específicos para futuros profesores de inglés incidieran en el desarrollo de un pensamiento crítico y reflexivo real. Para ello es imprescindible facilitarles el acceso al pensamiento de docentes en ejercicio y que puedan conocer de primera mano cómo se enfrentan estos a situaciones problemáticas. La elaboración de supuestos prácticos extraídos de documentos personales de este tipo y de situaciones hipotéticas basadas en la realidad del aula puede activar la reflexión dentro de procesos simulados de toma de decisiones.

Existen numerosas posibilidades de acceso al conocimiento práctico de los docentes en ejercicio utilizando las historias que ellos mismos nos cuentan. Una opción interesante sería la elaboración de un portafolio electrónico que fomente los procesos de metacognición y autorregulación del docente sobre el proceso de enseñanza-aprendizaje como medio para el desarrollo de sus competencias profesionales y mejora de su práctica en el aula (Esteve et al., 2008). También sería interesante, tal como propone Marcelo (2009), fomentar la creación de redes externas de profesores en formación y noveles como parte de un programa de inserción colaborativo en el que se les faciliten vías de comunicación e intercambio de información, por ejemplo mediante seminarios, grupos de trabajo o discusión de casos, que incentiven a su vez un proceso de reflexión y resolución conjunta de problemas, es decir lograr el desarrollo de un "interpensamiento colaborativo" tal como lo denominan Medina y Domínguez (1997).

Por último, existen múltiples posibilidades de investigación centrándose en aspectos concretos de la problemática descrita por los docentes. Pero quizá uno de los 
más interesantes es cómo ante estas situaciones de dificultad el profesor "transforma" sus teorías implícitas en práctica. Conocer cómo está interconectado el pensamiento teórico y el práctico y cómo se influyen mutuamente en su construcción y adaptación constante aportaría una información muy valiosa de cara a encontrar la mejor manera de presentar la teoría durante la formación inicial.

En síntesis, dado el gran vacío que existe en el estudio del pensamiento de los profesores de inglés como lengua extranjera, cualquier investigación futura basada en sus diarios de clase y relatos autobiográficos contribuirá de manera especial a conocer no sólo la práctica docente de manera directa sino también el proceso de construcción de la identidad y del conocimiento realista de los docentes.

\section{Bibliografía}

Alliaud, Andrea y Antelo, Estanislao (2009): "Iniciarse en la docencia. Los gajes del oficio de enseñar", Profesorado. Revista de Currículum y Formación del Profesorado, 13 (1), 89-100. http://www.ugr.es/ recfpro/rev131ART6.pdf [consulta: 23 marzo 2015].

Arnaus, Remei (1995): "Voces que cuentan y voces que interpretan: Reflexiones en torno a la autoría narrativa en una investigación etnográfica", en Déjame que te cuente. Ensayos sobre narrativa y educación, Larrosa, J. et al., Barcelona, Alertes, 62-78.

Bolívar, Antonio; Domingo, Segovia y Fernández, Manuel. (2001): La investigación biográfico-narrativa en educación. Enfoque y metodología, Madrid, La Muralla.

Bolívar, Antonio (2002): “¿De nobisipsissilemus? Epistemología de la investigación biográfico-narrativa en educación”, en Revista Electrónica de Investigación Educativa, 4 (1). http://redie.ens.uabc.mx/vol4no1/contenido-bolivar.html [consulta: 15 mayo de 2014].

Borg, Simon (2006): Teacher Cognition and Language Education, London, Continuum.

Cambra, Margarida y Palou, Juli (2007): "Creencias, representaciones y saberes de los profesores de lenguas en las nuevas situaciones plurilingües escolares de Cataluña", Cultura y Educación, 19 (2), 149-163.

Eirín, Raúl; García, Herminia María y Montero, Lourdes (2009): “Profesores principiantes e iniciación profesional. Estudio exploratorio", Profesorado. Revista de Currículum y Formación del Profesorado, 13 (1), 101-115. http://www.ugr.es/ recfpro/rev131ART7. pdf [consulta: 5 febrero de 2016].

Esteve, Olga et al. (2008): "La interrelación de contextos en la investigación en el aula: una aproximación ecológica”, en El proceso de enseñar lenguas. Investigaciones en Didáctica de la Lengua, Barrio, J. L. (coord.), Madrid, La Muralla, 135-167.

Esteve, Olga; Melief, Ko y Alsina, Ángel (coords.) (2010): Creando mi profesión. Una propuesta para el desarrollo profesional del profesorado, Barcelona, Octaedro.

Fernández, Manuel (2010): “Aproximación biográfico-narrativa a la investigación sobre formación docente", Profesorado. Revista de Currículum y Formación del Profesorado, 14 (3), 17-32. http://www.ugr.es/ recfpro/rev143ART1.pdf [consulta: 11 junio de 2015].

González, José Fernando; Álvarez, María Luisa y González, Julio Antonio (1991): "El profesor principiante. Análisis de sus problemas", Magíster, 9, 109-128.

González, Gustavo y Becerril, Raquel (2013): "El recorrido investigador de un educador novel explicado desde una perspectiva autobiográfica", Revista Electrónica Interuniversitaria de Formación del Profesorado, 3 (16), 61-78, doi: http://dx.doi.org/10.6018/reifop.16.3.186511 
Luchini, Pedro Luis (2007): "Lectura comprensiva: estudio de un caso con dos docentes de inglés”, Didáctica. Lengua y Literatura, 19, 135-153.

Marcelo García, Carlos (1987): El Pensamiento del profesor, Barcelona, Ceac.

Marcelo García, Carlos (1991): Aprender a Enseñar: un estudio sobre el proceso de socialización de profesores principiantes, Madrid, C.I.D.E.

Marcelo García, Carlos (1993): "El primer año de enseñanza. Análisis del proceso de socialización de profesores principiantes", Revista de Educación, (300), 225-277.

Marcelo García, Carlos (2009): "Los comienzos en la docencia: un profesorado con buenos principios", Profesorado. Revista de Currículum y Formación del Profesorado, 13 (1), $1-25$.

McDermott, Ray y Varenne, Hervé (2006): "Reconstructing culture in educational research", en Innovations in educational ethnography. Theory, methods, and results, Spindler, G. y Hammon, L., New Jersey, LEA, 3-32.

Medina, Antonio y Domínguez, Concepción (1997): “Análisis y evolución del conocimiento y creencias del profesorado: la construcción del conocimiento profesional en la última década", Publicaciones, 25-26-27, 497-531.

Melief, Ko; Tigchelaar, Anke y Korthagen, Fred (2010): “Aprender de la práctica”, en Creando mi profesión. Una propuesta para el desarrollo profesional del profesorado, Esteve, O; Melief, K. y Alsina, A., Barcelona, Octaedro, 19-37.

Plummer, Ken (1989): Los documentos personales. Introducción a los problemas y la bibliografía del método humanista, Madrid, Siglo XXI.

Porlán, Rafael y Martín, José (1991): El diario del profesor: un recurso para la investigación en el aula, Sevilla, Díada.

Ruiz, Raúl (2010): "El proceso de lectura en lengua extranjera: de la descodificación a la interpretación”, Didáctica. Lengua y Literatura, 22, 311-324.

Sandín, M. Paz (2003): Investigación cualitativa en educación. Fundamentos y tradiciones, Madrid, Mc Graw Hill.

Veenman, Simon (1984): "Perceived problems of beginning teachers", Review of Educational Research, 54, 143-178.

Vera Vila, Julio (1988): El profesor principiante (las dificultades de los profesores en los primeros años de trabajo en la enseñanza), Valencia, Promolibro.

Watson, Sue (2006): "The stories people tell: teaching narrative research methodology in New Zealand", en Narrative research on learning. Comparative and international perspectives, Trahar, S. et al., Oxford, Symposium Books, 61-75.

Zabalza, Miguel Ángel (1991): Los diarios de clase. Documentos para estudiar cualitativamente los dilemas prácticos de los profesores, Barcelona, PPU. 